\title{
Role of Physical Rehabilitation in Total Hip Arthroplasty with Neurological Disorder Patient: A Single Case Study
}

\author{
Amit Navani $^{1}$, Hardik Trivedi ${ }^{2}$ \\ (1) Assistant Professor, Shree K. K. Sheth Physiotherapy College, Rajkot, Gujarat \\ (2) Incharge Principal, Shree K. K. Sheth Physiotherapy College, Rajkot, Gujarat
}

DOI: 10.29322/IJSRP.12.01.2022.p12161

http://dx.doi.org/10.29322/IJSRP.12.01.2022.p12161

\begin{abstract}
Objective: To determine the effectiveness of physiotherapy treatment in total hip replacement with cerebral palsy patient.

Materials and Method: The study includes a patient of age 70 years with total hip replacement on right side, an exercise protocol was followed for physiotherapy treatment of total hip replacement and exercise was done on regular basis daily.

Result: In this study, pain assessment, improvement in range of motion and able to do activities of daily living after giving physiotherapy management was checked.

Conclusion: This study concluded that after receiving physiotherapy treatment in hip replacement with cerebral palsy case, have a significant effect in reducing pain, increasing range of motion and improving the performance in daily activities.
\end{abstract}

Index Terms- Total Hip Arthroplasty (THA), Cerebral Palsy, Rehabilitation, Functional Independence Measure.

\section{INTRODUCTION}

$\mathrm{T}$ his article guides a stepwise walkthrough by Experts for writing a successful journal or a research paper starting from inception of ideas till their publications. Research papers are highly recognized in scholar fraternity and form a core part of $\mathrm{PhD}$ curriculum. Research scholars publish their research work in leading journals to complete their grades. In addition, the published research work also provides a big weight-age to get admissions in reputed varsity. Now, here we enlist the proven steps to publish the research paper in a journal.

One of the most widely performed surgical interventions for joint disease of the hip and some fractures that compromise the vascular supply to the head of the femur is total hip arthroplasty. Total hip arthroplasty has been successfully performed since the early 1960s. Sir John Charnley, a surgeon from England, is credited with the initial research and clinical application of THA, which subsequently has evolved into contemporary hip arthroplasty. A variety of implant designs, materials, and surgical approaches have been developed and modified over the years since the early replacements. Today total hip implant systems typically are composed of an inert metal modular femoral component and a high-density polyethylene acetabular component ${ }^{1}$ There are several standard (conventional) approaches that may be used during traditional THA procedures: posterior (or postero-lateral), lateral, and anterior (or anterolateral). Each has its advantages and disadvantages. Traditionally, physiotherapy has been a routine component of patient rehabilitation following hip joint replacement. Due to the introduction of initiatives such as integrated care pathways, the length of hospital stay following joint replacement surgery has markedly and rapidly decreased, with the duration period for post-operative in-patient physiotherapy being reduced.

One of the more common musculoskeletal problems in the elderly is fracture of the hip or, more correctly, fracture of the most proximal portion of the femur in the hip joint area. More than $70 \%$ of hip fractures occur in individuals who are more than 70 years of age. In the elderly patient, displaced intracapsular fractures are typically managed with prosthetic replacement of the femoral head to avoid a relatively high incidence of non-union. Fracture neck femur is an orthopaedic emergency which need to be reduced or treated within 24 hours to get an optimum result. ${ }^{2}$ 
Cerebral palsy (CP) is the most common paediatric-onset physical disability, with a prevalence of 1.7-3.1 per 1000 live births in highincome countries and higher prevalence in low- income countries. Cerebral palsy is caused by an insult to or malformation of the developing brain that affects motor control centres and leads to alterations in growth and development. Although the brain lesion that causes $\mathrm{CP}$ is non-progressive, it affects overall health and especially mobility throughout the lifespan. In children and young adults with $\mathrm{CP}$, lower-limb muscle strength is closely related to independent mobility with muscle adaptations resulting in reduced walking speed and distance, as well as more frequent tripping and falling. Several factors underlie the loss of muscle mass and function with advanced age, such as anabolic resistance, impaired muscle quality, and nutritional factors. Apart from the decrease in muscle mass, which is reflected by a decrease of up to $50 \%$ in the size and number of muscle fibres from 20 to 90 years of age, there are also composition changes in the muscle that occur with age. Moreover, there is an age-dependent increase in muscle fat content, which is known to be positively correlated with whole-body fat. Age-related muscle fat infiltration can be part of the sarcopenia process, as it is associated with lower muscle strength and an increased risk of developing mobility limitations. ${ }^{3}$ The gross motor function measure (GMFM) is an observational clinical tool designed to evaluate change in gross motor function in children with cerebral palsy. The functional independence measure (FIM) includes 18 items designed to assess the amount of assistance required for a person with a disability to perform basic life activities safely and effectively. The activities include a minimum set of skills related to self-care, sphincter control, transfers, locomotion, communication, and social cognition. ${ }^{5}$

\section{PATIENT HISTORY}

This case relates to the assessment and initial management of a 70-year old male, presented with the operated case of Intertrochanteric femur fracture on right side. Mechanism of injury was domestic fall during walking and more weight was on right side. Patient was admitted, after doing proper investigation Intertrochanteric fracture of femur bone on right side was found and he was operated with total hip arthroplasty on next day. Again after some days due to some circumstances patient kept its right leg in internal rotated position, so there was hip dislocation on operated side. And re-operation was done for the same. Before injury also, patient had a supported walking because of weakness related to cerebral palsy. His chief complaint was pain at right hip region, aggravating factor was movement of right leg along with the difficulty to lift right leg, unable to walk or go anywhere; even functional activities was also affected. He was only able to sit at the edge of the bed with support. He did not have any complaint of other joint pain or stiffness in the joint. No relevant or significant past medical history was described, no regular medication was taken and no known drug allergies were noted. He also denied any respiratory distress, history of smoking or alcohol intake. He had been off work since long due to independent walking

\section{EXAMINATION}

On examination, the patients vital were normal had and a forward head posture was observed on sitting. Pain assessment was taken at initial stage before starting the physiotherapy sessions on Visual Analog Scale (VAS), the score was $\mathbf{5}$, and type was dull aching pain. Weight bearing position elevates the pain intensity. Scar was present on lateral aspect of right hip region, and the skin texture was normal. The active Range of motion (ROM) was affected in right hip joint, and spinal mobility (flexion and extension) was also limited. The active range was limited as compared to passive due to weakness found in right hip musculature. The strength in hip joint and knee joint muscles was reduced as compared to sound side. Sensory examination (superficial and deep) was done. Dermatome pattern was normal no atrophy of gluteus or quadriceps muscles was observed. GMFM score was measured to evaluate the gross motor function. Patient had more affection in his functional work so Functional independence measure score was taken to measure the independent level of patient, FIM score was $\mathbf{6 1}$ during examination.

\section{MANAGEMENT}

Many treatments have been advocated for total hip arthroplasty. The existence of so many different treatments, each with its own group of enthusiastic supporters, suggests that no single treatment is unequivocally superior to others. The fundamental goal of treatment is to restore and maintain function. Many studies have included early mobilization, gentle range of motion exercises, and weight bearing should be part of managing all patients with total hip arthroplasty. Exercise should be two types stretching exercises to prevent further loss of range of motion and promote faster return to normal range, and strengthening. While all post-operative rehabilitation programs should address common strength and range of motion impairments after surgery. ${ }^{6} \mathrm{~A}$ physiotherapist's most useful role is in patient education and instruction in appropriate exercise to prevent loss of further range and strength. ${ }^{4}$ Among all the above treatments physiotherapy play a significant role in the treatment of total hip arthroplasty. After through assessment physiotherapy rehabilitation protocol was given to the patients. The goal of rehabilitation was to optimize a patient's postoperative level of function. The patient was permitted to bear as much weight as tolerated almost immediately after surgery. A number of factors affect the extent and duration of postoperative weight-bearing restrictions and the need for an ambulation aid during transfers, walking, and ascending and descending 
stairs. There are also potential benefits of safe levels of early weight bearing after THA, specifically the reduction of bone demineralization from decreased weight bearing and the earlier recovery of functional mobility. The protocol was as follows;

\section{DAY 01-14}

The emphasis of this phase rehabilitation after a standard surgical approach is on patient education to reduce the risk of early postoperative complications, in particular dislocation of the operated hip.

- Ankle pumping exercise to prevent venous stasis, thrombus formation

- Patient and caregiver education about motion restrictions, safe bed mobility, transfers, and precautions during other ADL

- While in bed, active-assistive (A-AROM) exercises of the hip within protected ranges.

- Sub maximal muscle-setting exercises of the quadriceps, hip extensor, and hip abductor muscles, to elicit a muscle contraction

- Activities that involve standing on the operated extremity and rotating away from the involved side and hip flexion more than $90^{\circ}$ were avoided.

- Heel sliding exercise (Hip Flexion up to $60^{\circ}$ )

- Straight leg raise

Exercise was done with 15 repetitions, with 2 set each.

\section{DAY 15-28}

- Exercise was same but the repetition was increased. Emphasize increasing the number of repetitions rather than the resistance to improve muscular endurance.

- Closed-chain hip flexion and extension, placing only the allowable amount of weight on the operated extremity.

- Patient education continues throughout these phases of rehabilitation in preparation for a return to anticipated activities in the home.

- Supported walking was started with the help of family members

- Active resisted exercise for quadriceps and abductors was given

- Heel slide was increased up to $90^{\circ}$

- Integrate posture training during ambulation, emphasizing an erect trunk, vertical alignment, equal step lengths, and a neutral symmetrical position of the legs.

Exercise was done with 20 repetitions, with 2 set each.

\section{DAY 29-45}

- Intensity of exercise was increased along with the repetition.

- Resisted exercise of hip and knee musculature was given.

- Resisted exercise for abductor muscles.

- $\quad$ Sit to stand exercise was performed with support.

- $\quad$ Steps up and down with support.

- Progressively increase the length of time and distance of a low-intensity walking program of 5 days a week. Exercise was done with 20 repetitions, of $1 \mathrm{~kg}$ and $2 \mathrm{~kg}$ weight, with 2 set each.

\section{RESULT}

FIM outcome score was checked after physiotherapy treatment and score was increased by 99 out of

126. This indicates patient does its work independently with some assistance or self. The active hip range strength was increased on right side. The VAS score was also taken after physiotherapy treatment and it was decreased. The patient had achieved supported walking. 


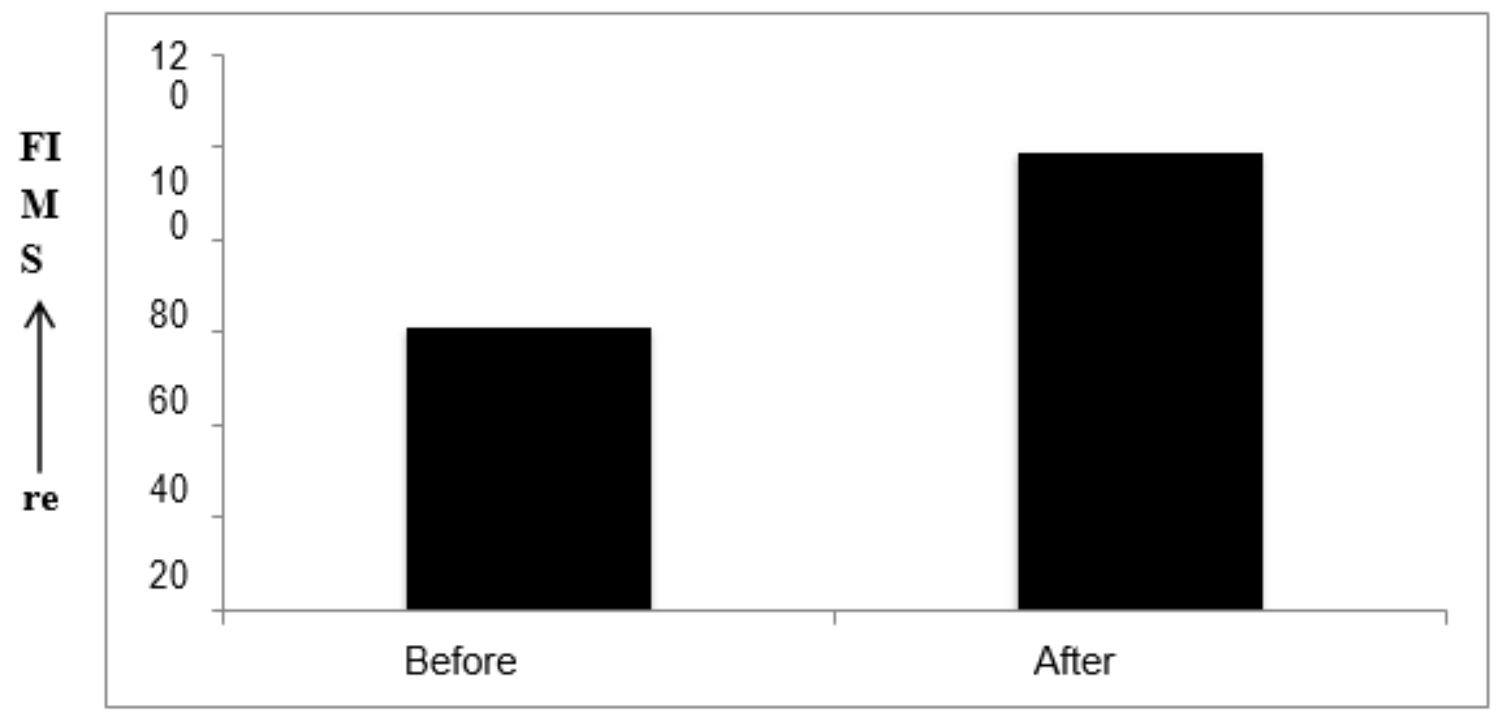

Treatment

Figure 1.1- Indicate the FIM score before and after the exercise

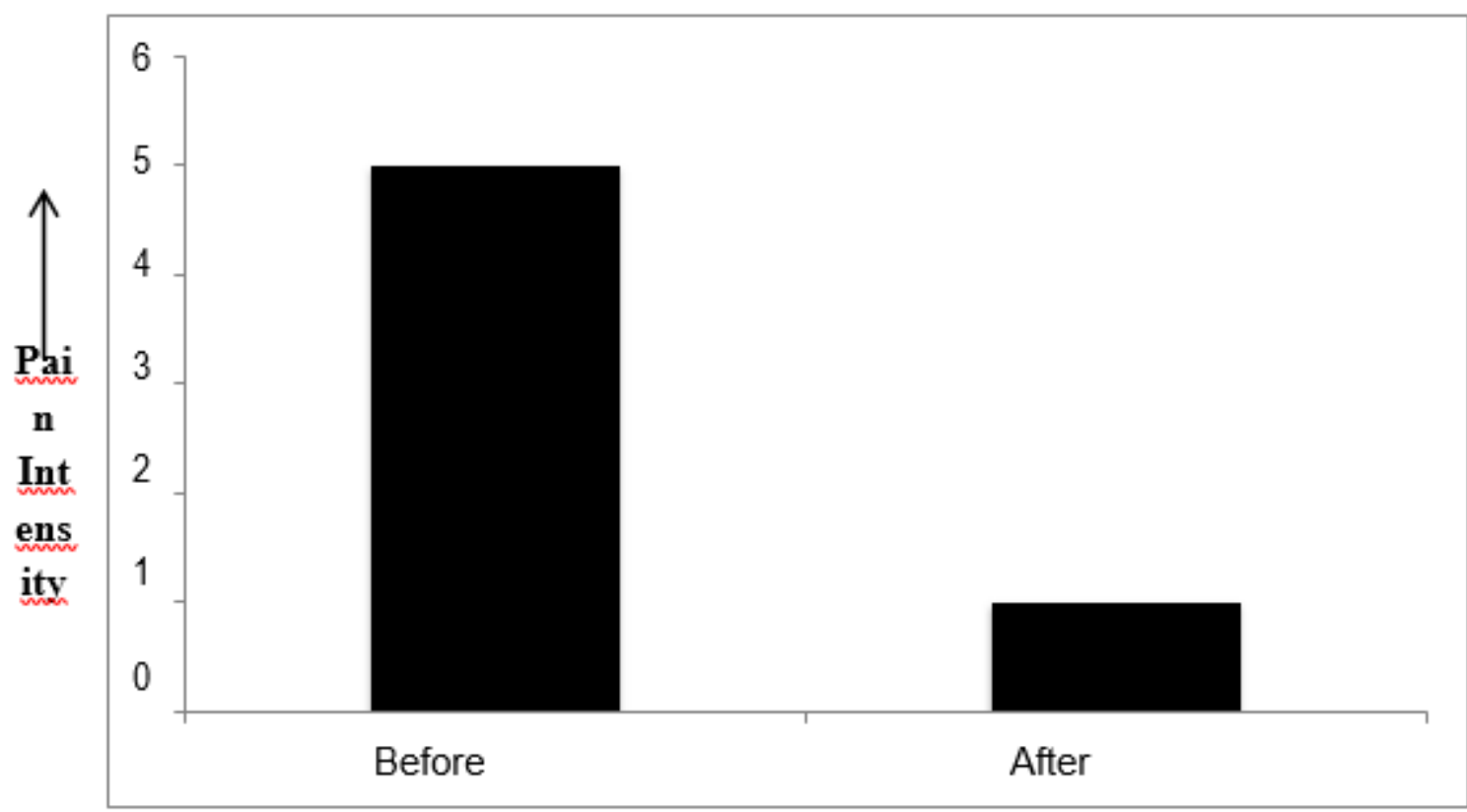

Treatment

Figure 1.2- Indicate amount of pain intensity before and after exercise

\section{DISCUSSION}

The study finds that it is possible to establish the extent to which post discharge physiotherapy exercise is effective, in terms of improving function, quality of life, mobility, range of hip joint motion and muscle strength, for unilateral total hip arthroplasty. Julia R. D'Agostino conclude regular exercise will lead to improvements in overall quality of life and physical health of an individual with 
cerebral palsy. ${ }^{8}$ This physical therapy protocol, which was focused on reducing supervised visits early after THA and retraining higher level activities later in the course of recovery, had a positive effect on outcomes without compromising safety. This study was envisioned with timing and content of this protocol to motivated or return the patient in daily routine activities. An important component of successful management of any condition is educating patients and informing them about the planned treatment. Objectives of physiotherapy and rehabilitation applications in patients with THR are to prevent disability, improve mobility, to increase functional capacity, and to provide pain relief. In this study the patient had weakness previously because of cerebral palsy and was having supported walking. After operation patient had weakness in operated side and was not able to walk, but after proper physiotherapy session he was able to walk with support or assistive device. Patient confidence was gained in walking as it was before operation. The functional activities were started for example using washroom, bathing, to self-do its own activities. There was also change in GMFM score in pre and post-operative physical rehabilitation and it indicate change in term of improvement in daily task. In view of her previous response to treatment, he was very happy with the progress he had made during the 6-week period. As the weakness was more due to neurological involvement but exercise protocol helps the patients to gain the strength and mobility as it was before operation. He felt confident in returning to daily routine activities.

\section{CONCLUSION}

The results of this study indicate that a treatment timing and focus on return to daily activity in cerebral palsy patients results in improvements in function and biomechanics after THA, and is feasible and safe to complete. The study concludes that physiotherapy plays an important role in treatment of total hip replacement.

\section{ACKNOWLEDGEMENT}

The author is thankful to subject who participated in this study to carry out this work.

\section{CONFLICT OF INTEREST}

There is no personal or institutional conflict of interest for this study

\section{REFERENCES}

[1] Carolyn Kisner, Lynn allen Colby, 5th edition; Therapeutic Exercise foundations and techniques.

[2] John Ebnezar; Essential of orthopaedics for physiotherapy; 2nd edition.

[3] Olaf Verschuren1, Ana R.P. Smorenburg et al;Determinants of muscle preservation in individuals with cerebral palsy across the lifespan: a narrative review of the literature; Journal of Cachexia, Sarcopenia and Muscle 2018; 9: 453-464.

[4] Punia Sonu, Sushma et al; Effect of physiotherapy treatment on frozen shoulder: a case study, Indian Journal of Physiotherapy and Occupational Therapy. JanuaryMarch 2015, Vol. 9, No. 1.

[5] Kenneth J. Ottenbacher, Yungwen Hsu et al; The Reliability of the Functional Independence Measure: A Quantitative Review; Arch Phys Med Rehabil Vol77; December 1996.

[6] Kathleen C. Madara et al; Progressive rehabilitation after total hip arthroplasty: a pilot and feasibility study, The International Journal of Sports Physical Therapy; Volumw 14; Number 4;August 2019;Page 564.

[7] Madawi Alotaibi et al; The efficacy of GMFM-88 and GMFM-66 to detect changes in gross motor function in children with cerebral palsy (CP): a literature review; Disability and rehabilitation; 36 (8); 617-627; 2014.

[8] Julia R. D'Agostino; Effect of Physical Activity on Perceived Quality of Life of an Individual with Cerebral Palsy; Honors Research Projects; 138.

\section{AUTHORS}

First Author - Amit Navani, Assistant Professor, Incharge Principal, Shree K. K. Sheth Physiotherapy College, Rajkot, Gujarat Second Author - Hardik Trivedi, Incharge Principal, Shree K. K. Sheth Physiotherapy College, Rajkot, Gujarat 